\title{
A Multi-Scale Multi-Physics Modeling Framework of Laser Powder Bed Fusion Additive Manufacturing Process
}

Jing Zhang ${ }^{1}$, Yi Zhang ${ }^{1}$, Weng Hoh Lee ${ }^{1}$, Linmin Wu ${ }^{1}$, Sugrim Sagar ${ }^{1}$, Lingbin Meng ${ }^{1}$, HyunHee Choi ${ }^{2}$, Yeon-Gil Jung ${ }^{2}$

${ }^{1}$ Department of Mechanical and Energy Engineering, Indiana University-Purdue University Indianapolis, Indianapolis, IN 46202, USA

${ }^{2}$ School of Materials Science and Engineering, Changwon National University, Changwon, Gyeongnam 641-773, Republic of Korea

\begin{abstract}
A longstanding challenge is to optimize additive manufacturing (AM) process in order to reduce AM component failure due to excessive distortion and cracking. To address this challenge, a multi-scale physics-based modeling framework is presented to understand the interrelationship between AM processing parameters and resulting properties. In particular, a multi-scale approach, spanning from atomic, particle, to component levels, is employed. The simulations of sintered material show that sintered particles have lower mechanical strengths than the bulk metal because of their porous structures. Higher heating rate leads to a higher mechanical strength due to accelerated sintering rates. The average temperature in the powder bed increases with higher laser power. The predicted distortion due to residual stress in the AM fabricate
\end{abstract}

This is the author's manuscript of the article published in final edited form as:

Zhang, J., Zhang, Y., Lee, W. H., Wu, L., Choi, H. H., \& Jung, Y. G. (2018). A multi-scale multi-physics modeling framework of laser powder bed fusion additive manufacturing process. Metal Powder Report, 73(3), 151-157. https://doi.org/10.1016/j.mprp.2018.01.003 
component is in good agreement with experimental measurements. In summary, the model framework provides a design tool to optimize the metal based additive manufacturing process.

\section{Introduction}

Laser Powder Bed Fusion (L-PBF) is a primary additive manufacturing process for metallic components. A longstanding challenge is to optimize additive manufacturing process in order to reduce AM component failure due to excessive distortion and cracking. The growth need of reliable methods to improve quality of AM parts greatly depends on the quantitative understanding of powder deposition and laser sintering during the 3D printing process. Although there are extensive experimental efforts and several modeling techniques for a variety of metallic materials, there is still a lack of thorough comprehensive understanding of the process-property relations in the PBF process, which hinders the wide application of the technique.

Modeling the PBF process requires a good level of understanding of the fundamental sciences, including heat transfer, powder mechanics, and modeling of laser source, among others. The sintering process usually occurs very fast (in nano seconds), that extremely increases the difficulty of experimental measurements. Molecular dynamics (MD) simulation is a powerful tool that is capable to capture the sintering kinetics at very short time and nanosize scale. Discrete element modeling (DEM) is suitable to obtain the information at particle level, e.g., powder flow during the laser heating, as well as the powder movement during the recoating process. With the DEM simulation, localized powder size distribution and the corresponding powder size based properties can also be applied to the continuum FE model for complex-shaped components. 
In this work, a modeling framework of modeling the PBF process is proposed, as illustrated in Figure 1. Several length scales and multiple physics are included in this framework. Sintering mechanisms, atomic diffusion, and resultant mechanical properties are investigated using a molecular dynamics model at atomistic scale. Powder deposition, powder flow, and laser heating of the powder bed are simulated by a discrete element model at mesoscale. Additionally, a finite element study of thermal stress or distortion evolution at macroscale level is presented. The coupling between the different scales is also illustrated in Figure 1.

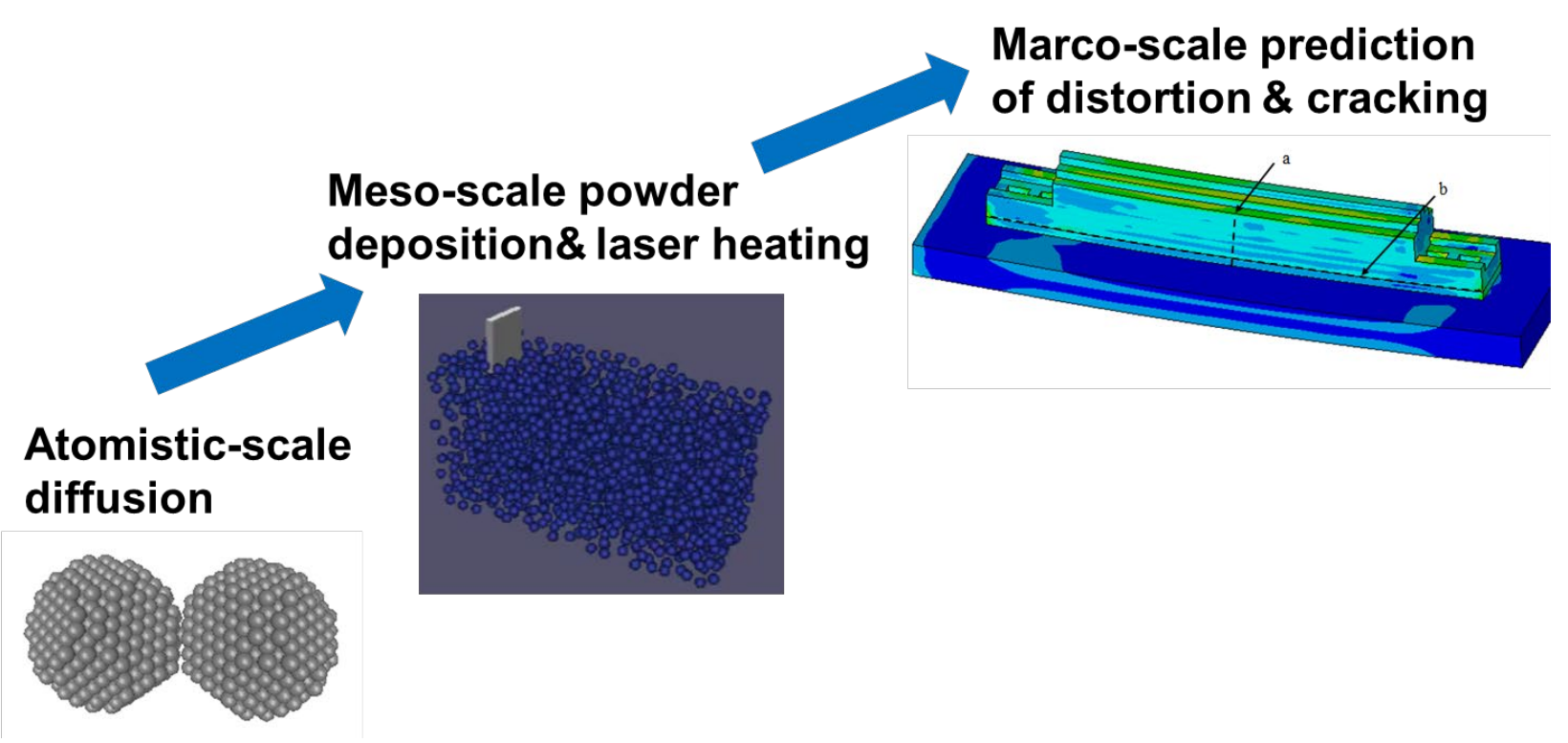

(a) 


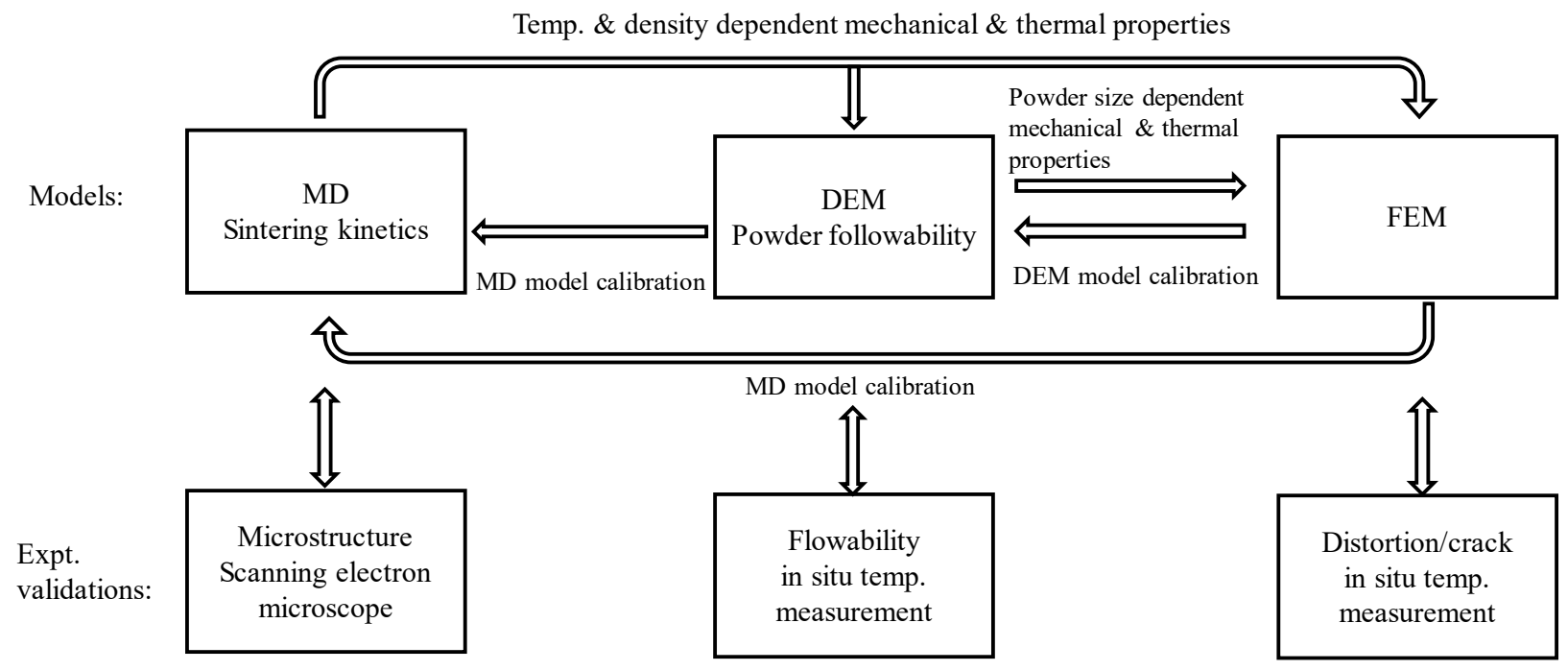

(b)

Figure 1: (a) Multi-scale multi-physics modeling framework of the laser powder bed fusion process, (b) Coupling between different scales and experimental validation.

\section{Methods}

\subsection{Molecular dynamics model}

The embedded-atom method (EAM) $[1,2]$ was used for describing the atomic interactions between nickel particles. In EAM, the total energy $E i$ of an atom $i$ is given by

$$
E_{i}=F_{\alpha}\left(\sum_{j \neq i} \rho_{\beta}\left(r_{i j}\right)\right)+\frac{1}{2} \sum_{j \neq i} \emptyset_{\alpha \beta}\left(r_{i j}\right)
$$

where $F$ is the embedding energy that represents the energy required to place atom $i$ of type $\alpha$ into the electron cloud. $\rho_{\beta}$ is the contribution to the electron charge density from atom $j$ of type $\beta$ 
at the location of atom $i, \phi_{\alpha \beta}$ is a pair-wise potential interaction, and $\alpha$ and $\beta$ are the element types (both were $\mathrm{Ni}$ in this work) of atoms $i$ and $j, r_{i j}$ is the distance between atoms $i$ and $j$. The multi-body nature of the EAM potential is a result of the embedding energy term. Both summations in the formula are over all neighbors $j$ of atom $i$ within the cutoff distance. In this work, molecular dynamics software package Large-scale Atomic/Molecular Massively Parallel Simulator (LAMMPS) [3] was used for simulations of sintering of nickel powder and also the mechanical behavior of sintered parts. Additional details of the MD model are given in Refs.[4, 5].

\subsection{Discrete element model}

Discrete element method (DEM) is a numerical technique that calculates the interaction of a large number of particles [6], which is particular useful to describe the L-PBF process, including powder flow and powder heating. In this study, the LIGGGTS package [7] was used to simulate the interaction among metal particles. For particle flow simulations, the DEM calculates the defined displacements and rotations of discrete bodies of various types of particle shapes, which can be predicted through the gathering of assembled particles [8]. Particles are simulated through solving the Newton's second law of motion and rigid body dynamics equation combined with specific time-stepping algorithms [9, 10]. Equations 2 and 3 are the corresponding governing equations for translational and rotational motion, respectively [10]:

$$
\begin{gathered}
m_{i} \ddot{x}_{i}=m_{i} g+\sum_{j} F_{i j} \\
I_{i} \ddot{\theta}=\sum_{i}\left(r_{i j} \cdot F_{i j}\right)
\end{gathered}
$$


with $\ddot{x}_{l}$ translational acceleration, $m_{i}$ mass of the particles $i, g$ acceleration due to gravity, $F_{i j}$ force at contact with neighboring particles $j, r_{i j}$ vector directed from the center of the particle $i$ to the contact point with particle $j$, and $I_{i}$ the mass moment of inertia of the particle $i$.

For inter-particle interactions, Hertzian potential force with no cohesion reaction is used. The Hertzian formula to compute the pair potential forces are as follows [11-13]:

$$
\begin{gathered}
F_{h k}=\left(k_{n} \delta n_{i j}-m_{e f f} \gamma_{n} v_{n}\right)-\left(k_{t} \Delta s_{t}+m_{e f f} \gamma_{t} v_{t}\right) \\
F_{h z}=\sqrt{\delta} \sqrt{\frac{R_{i} R_{i}}{R_{i}+R_{j}}} F_{h k}=\sqrt{\delta} \sqrt{\frac{R_{i} R_{i}}{R_{i}+R_{j}}}\left[\left(k_{n} \delta n_{i j}-m_{e f f} \gamma_{n} v_{n}\right)-\left(k_{t} \Delta s_{t}+m_{e f f} \gamma_{t} v_{t}\right)\right]
\end{gathered}
$$

with $R_{i}$ and $R_{j}$ are the radii of particle $i$ and $j$, respectively, $\delta$ the overlap distance of two particles, $k$ the elastic constant, $\gamma$ the viscoelastic damping constant, $\Delta s$ the displacement vector between the two spherical particles which is truncated to satisfy a frictional yield criterion, $n_{i j}$ the unit vector along the line connecting the centers of the two particles, $v$ the component of the relative velocity of the two particles; indices $n$ and $t$ referring to normal and tangential contact respectively. $F_{h k}$ the force calculated using the Hookean style, $F_{h z}$ the force calculated using the Hertzian style, and $m_{\text {eff }}$ the effective mass of two particles. Additional details of the DEM are given in Refs. [4, 5]. 


\subsection{Finite element model}

A coupled thermal-mechanical finite element (FE) model including both thermal and mechanical components was developed. In this model, the temperature field is obtained from a transient heat transfer model for laser heating, and then used as the boundary condition of a quasi-static solid mechanical model to predict residual stress and distortion.

In the heat transfer model, the temperature field under selectively laser heating is obtained from the transient heat conduction equation:

$$
\rho\left(\frac{\partial H}{\partial t}\right)=-\nabla \cdot \boldsymbol{q}+Q
$$

where $\rho$ is the density of material, $H$ is the enthalpy, $t$ is time, Q is the heat generation rate, $\nabla$ is the gradient operator, and $q$ is the heat flux, which is a function of temperature and thermal conductivity by the Fourier's law:

$$
\boldsymbol{q}=-k \nabla T
$$

where $k$ is the temperature dependent thermal conductivity and $T$ is temperature.

Phase change, including melting and solidification during the laser heating is also considered in the heat transfer model. The latent heat is represented in the temperature dependent enthalpy by a sudden increase around the melting temperature. Heat convection is applied at the outer surface, according to the Newton's law of cooling:

$$
\frac{\partial Q}{\partial t}=h A\left(T_{s}-T_{\infty}\right)
$$

where $h$ is the convection coefficient, $A$ is the surface area, $T_{S}$ is the surface temperature and $T_{\infty}$ is the ambient chamber temperature. Additional details of the FE model are given in Refs [14, $15]$. 


\section{Results and discussion}

\subsection{MD modeling of sintering of nickel particles}

Figure 2 shows the diffusivities (D) at five different sintering temperatures, $700 \mathrm{~K}, 800 \mathrm{~K}, 900 \mathrm{~K}$, $1000 \mathrm{~K}$, and $1100 \mathrm{~K}$, which were calculated based on the mean-squared displacement (MSD). As expected, the diffusivities increase with increasing temperature for both the in (particle core) and out (particle shell) regions. The out region has a higher diffusivity than that of in region due to combined surface and grain boundary diffusions, while the in region has less diffusion due to mainly volume or lattice diffusions.

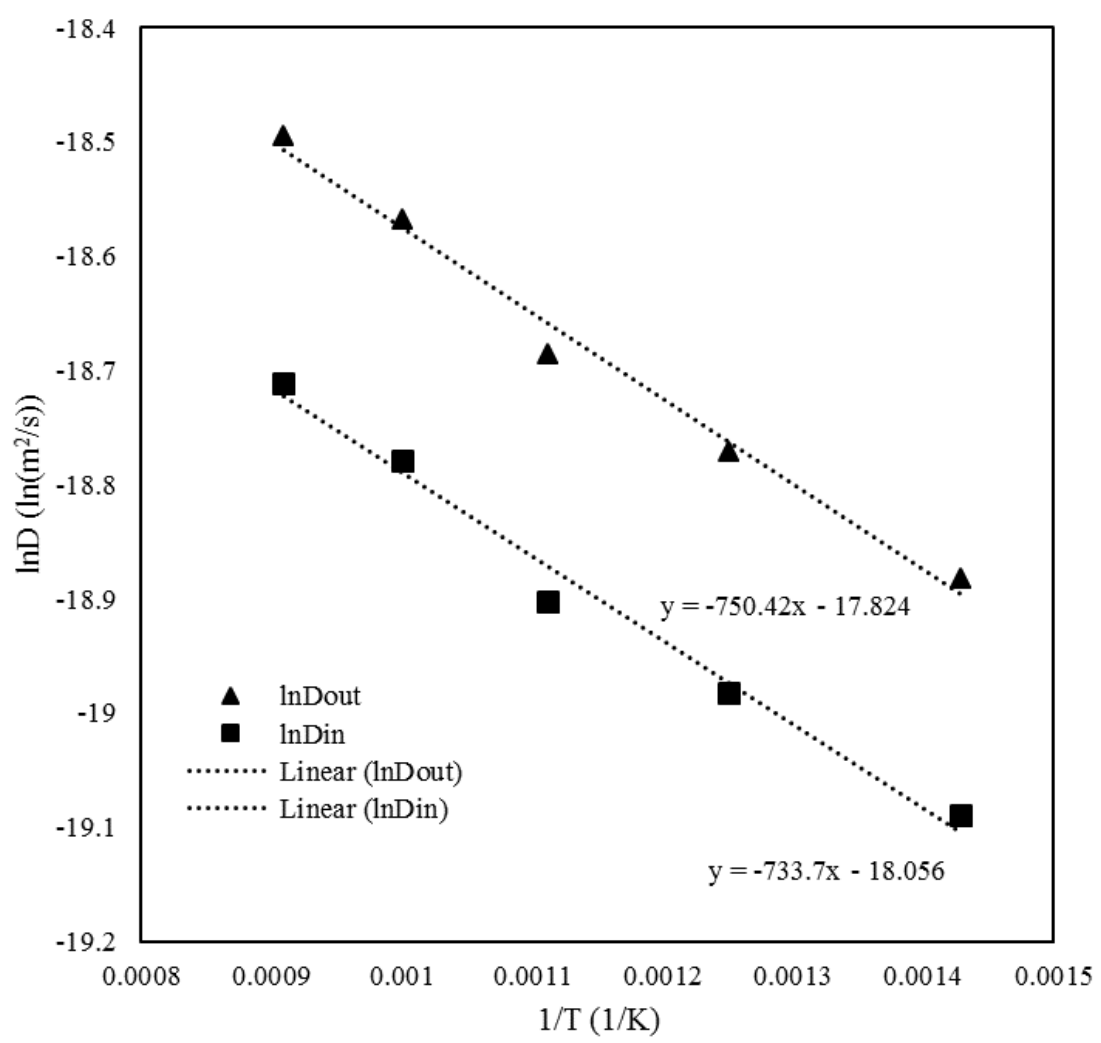


Figure 2: Diffusivities of in (particle core) and out (particle shell) regions at the temperature range of $700 \mathrm{~K} \sim 1100 \mathrm{~K}$. The linearly fitted lines and corresponding regression equations are also shown.

In order to understand the diffusion mechanisms during sintering, we compared the activation energy of particle surface (surface diffusion) and particle core (volume diffusion). The calculated activation energies of $\mathrm{Ni}$ diffusion is $6.10 \mathrm{KJ} / \mathrm{mole}$ in the particle core, and $6.24 \mathrm{KJ} / \mathrm{mole}$ on the particle surface, which are than the experimentally reported value $7.89 \mathrm{KJ} /$ mole [16].

Surface diffusion and volume diffusion have similar activation energy, suggesting the mass transfer in our model can't be simply explained by only one single dominating diffusion mechanism. Movements of atoms are mostly driven by crystal structure transformation rather than any conventional diffusion mentioned above. As shown in Figure 3a, the vector plot of atomic displacements illuminates the atom movements during sintering. By analyzing movement of atoms, neither surface diffusion nor grain boundary diffusion pattern can be identified. Atoms move in relatively small amount to change the particle's internal structure. At the end of sintering, different dislocation-free twinning structures are observed, as shown in Figures $3 \mathrm{~b}-3 \mathrm{~d}$. The color shown in the figures is defined by localized common neighbor analysis, where green color represents face-centered cubic (FCC), and red represents hexagonal close packed (HCP). The sintered structures at lower temperatures (700-1100 K) result in almost a single crystal FCC structure. For sintering at temperature of $1200 \mathrm{~K}$, the sintered structure ends up with a five-fold twinning structure. Sintering at $1300 \mathrm{~K}$ generates two parallel twin boundaries as shown in Figure $3 \mathrm{~d}$. Thus, the model suggests that the mass transfer in the MD model is primarily due to the dislocation-free elastic wave. 


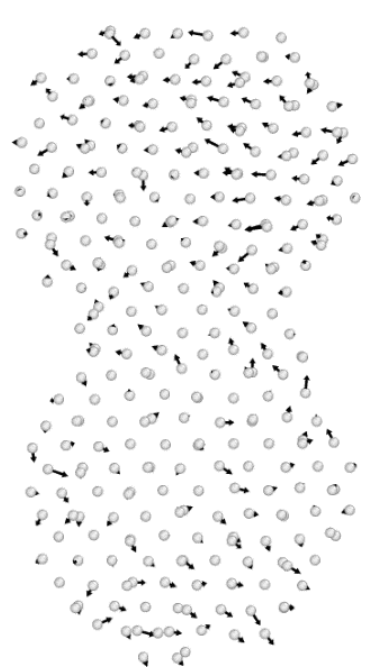

(a)

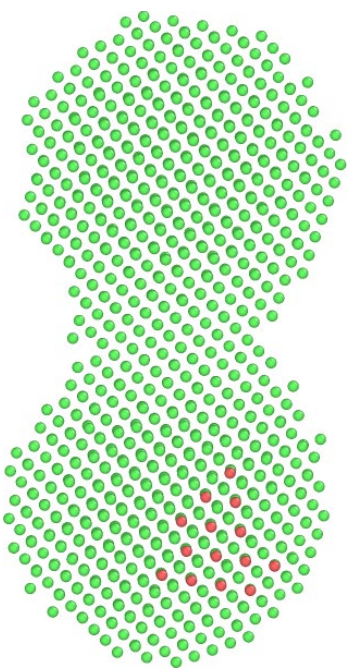

(b)

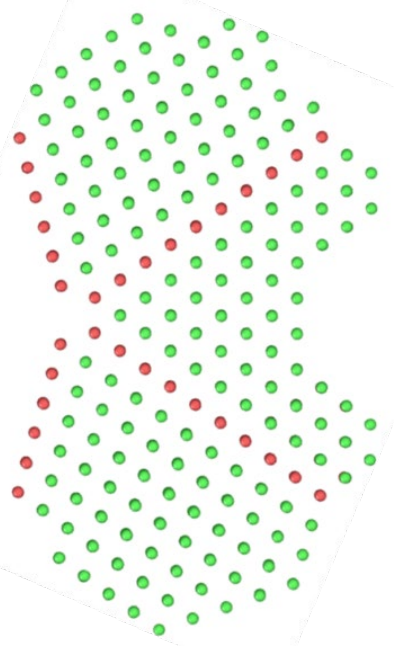

(c)

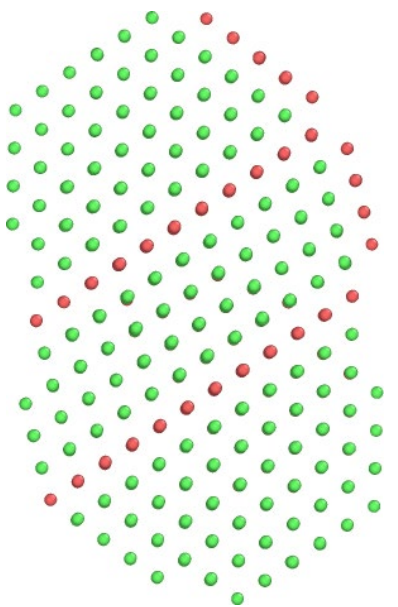

(d)

Figure 3: (a) Displacement vector plot during the sintering. The sintered structures with following sintering temperatures: (b) $1000 \mathrm{~K}$, (c) $1200 \mathrm{~K}$, and (d) $1300 \mathrm{~K}$.

\subsection{MD modeling of mechanical response of sintered nickel particles}

For the MD tensile test simulations, a tensile strain was applied in the direction of along the two particle centers. The pressures of the simulation systems were extracted, and the stress data were plotted against strain from 0 to 0.2 . Dislocation and common neighbor analysis were conducted to analyze the structural change during the tensile process as shown in Figure 4. The color curves in the figure show the dislocation lines based on localized structure [17]. The two-particle model's plastic deformation began at the strain of 0.10 , as shown in Figure 4a of initial occurrence of dislocations. In Figure $4 \mathrm{~b}$, the number of dislocation segments increased rapidly during the strain range of $0.10-0.11$, with a strong atomic motions along dislocation lines, indicating large plastic deformation. With further application of strain, the dislocations were annihilated by combining dislocation lines as shown in Figure 4c. 


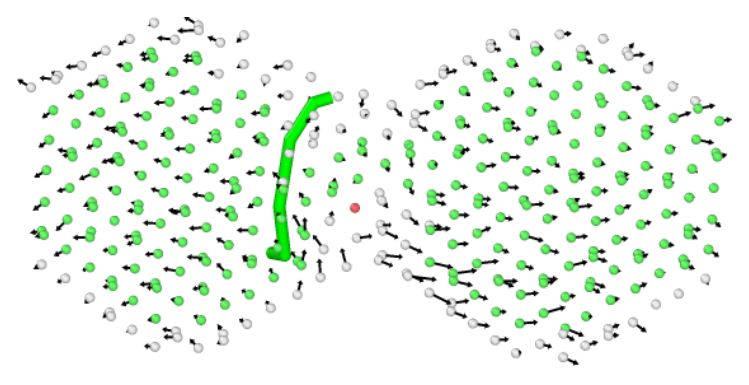

(a)

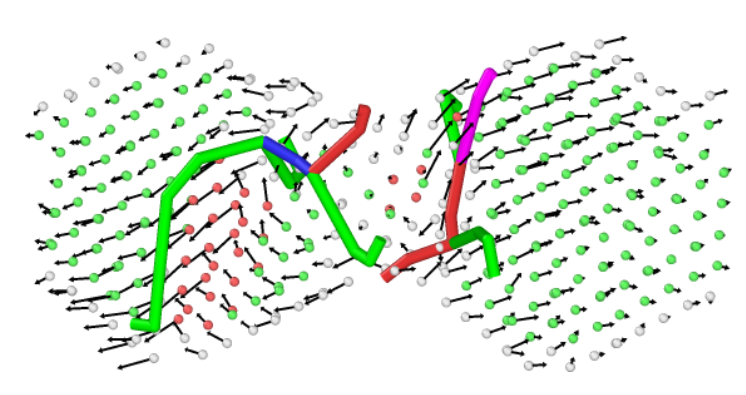

(b)

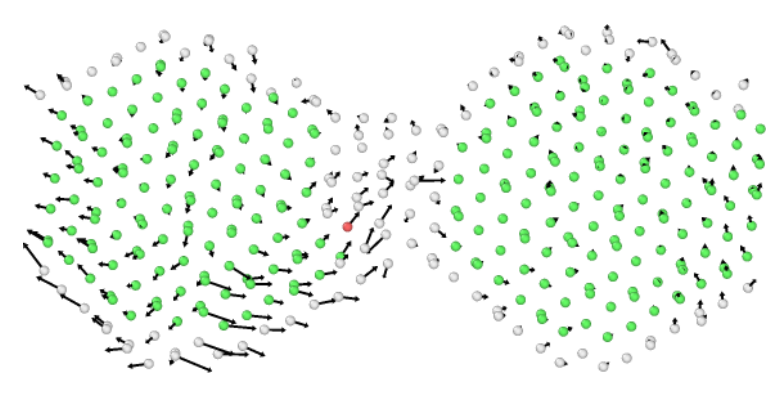

(c)

Figure 4: Structural change of the two-particle model under tensile load at various strains: (a) 0.10 , (b) 0.11 , and (c) 0.20 . Colors show the dislocation type: blue is $1 / 2<110>$ perfect, green is $1 / 6<112>$ Shockley, purple is $1 / 6<110>$ stair-rod, and red is other. 
Figure 5 shows the stress-strain curves of the all cases with different heating rates, including the two-particle model, the 41-particle model, and FCC bulk nickel crystal for comparison. At lower strain range, all the models had linear increments of stress, until the yield stress was reached at the strain range of $0.05 \sim 0.1$. Comparing the results, the sintered 41-particle structures had lower mechanical strengths than the bulk crystal, due to their porous structure and non-periodic nature of the nickel atoms. The two-particle model showed the lowest strength among all models, due to the small neck size between two nickel particles. By comparing the sintered structures at different heating rates, the model with a higher heating rate showed a higher mechanical strength. The reason is that during the same sintering period, higher heating rates promote densification to reduce porosity, and resultant density is more close to the bulk structure. Oscillations of the stress-strain curves after yield were observed in Figure 5. Due to the finite volume of the simulation system, the calculated stresses after yield are greatly affected by the increased porosity and the cut-off distance used in the EAM potentials.

The bulk Ni simulation in Figure 5 shows a calculated Young's modulus of 127 GPa, which is lower than the reported $200 \mathrm{GPa}$ in polycrystalline FCC Ni [18]. The underestimation is likely due to the accuracy of EAM potential used in this work. 


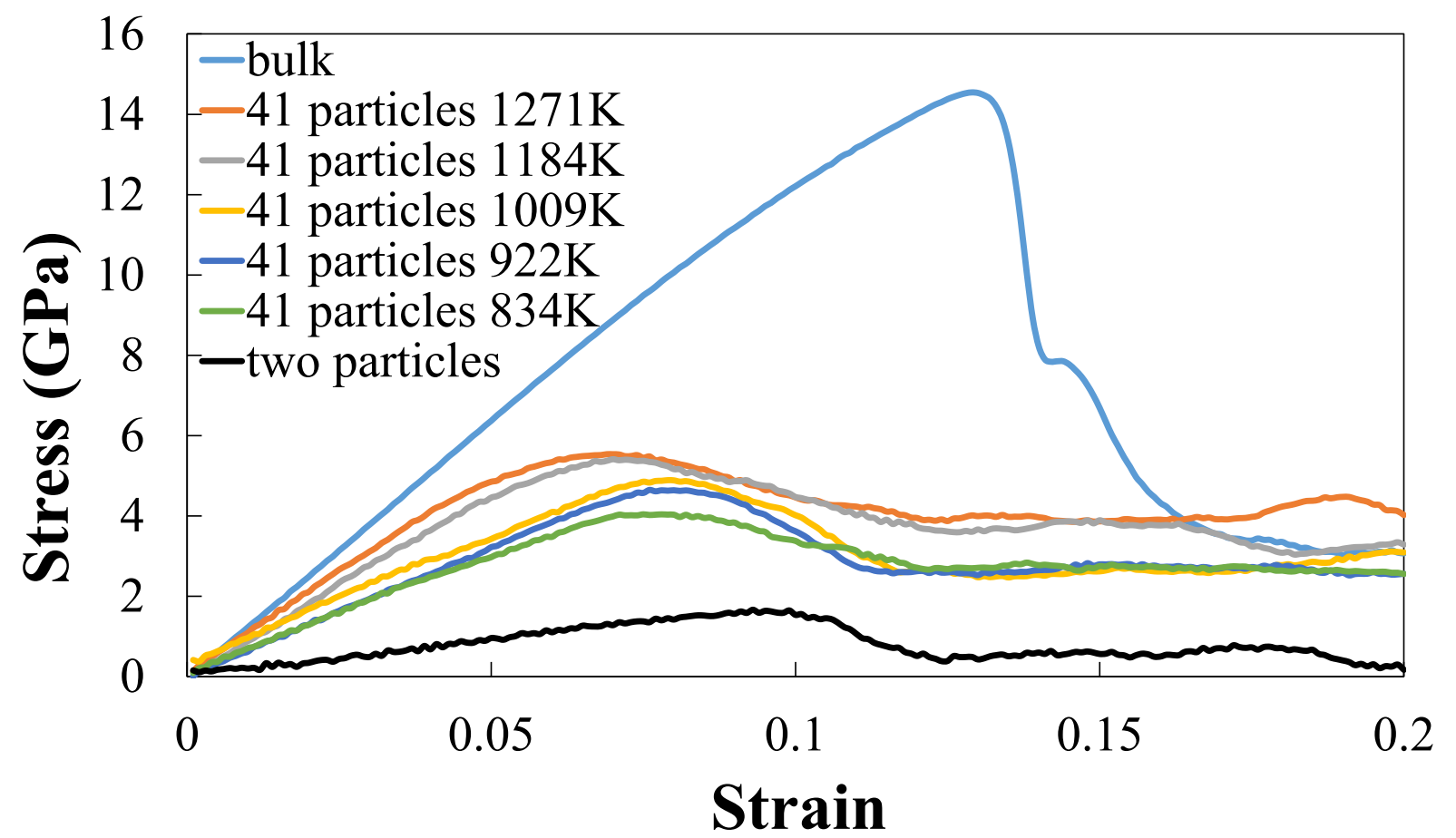

Figure 5: Stress vs. strain curves of sintered nickel particles at various heating rates.

\subsection{DEM of the sequence and temperature fields in L-PBF process}

Figure 6 shows the sequential schematics of the simulated L-PBF process. Four stages, i.e., powder deposition, recoating, laser heating, and holding period, were simulated. The particles in red have higher temperatures, while the particles in blue have lower temperature values.

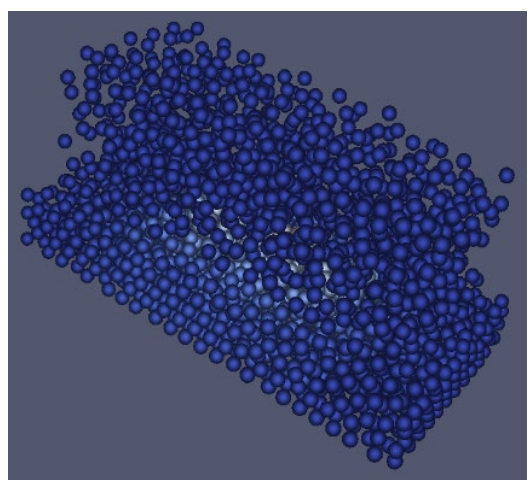

(a)

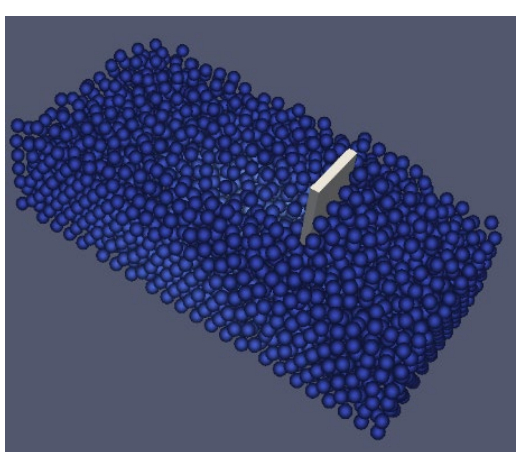

(b) 


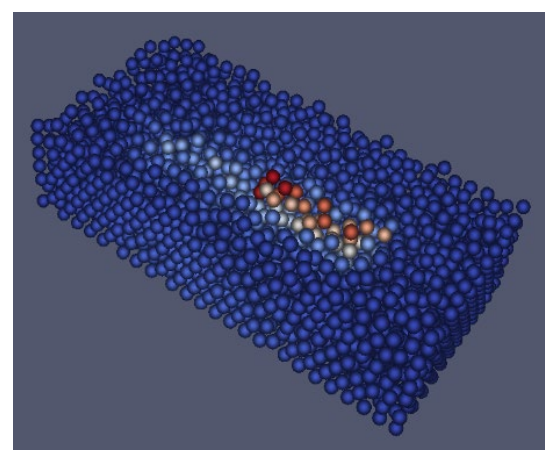

(c)
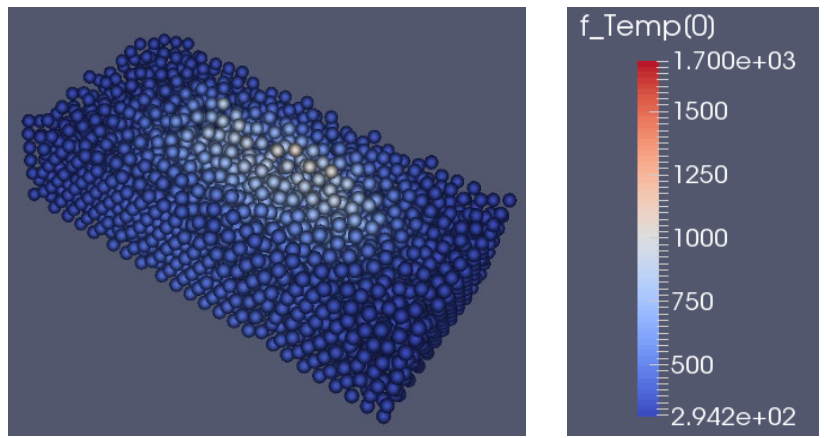

(d)

Figure 6: Temperature distributions in the L-PBF process simulations. (a) deposition of particles, (b) recoating process, (c)laser heating of particles, and (d) holding period. The particles are labeled using colors to present their temperature (unit: K).

Additionally, the effects of laser heating parameters, which includes laser power, scan speed, and hatch spacing, on the temperature distribution in the powder bed were analyzed. The temperature distributions are visualized, and the average temperature of the powder bed versus time are plotted to understand the behavior of thermal history. As an example, the average temperatures in the powder bed of $100 \mathrm{~W}$ and $300 \mathrm{~W}$ are plotted in Figure 7. The maximum average temperature produced by the $300 \mathrm{~W}$ laser can reach up to $383 \mathrm{~K}$ whereas the $100 \mathrm{~W}$ laser only reaches up to $358 \mathrm{~K}$. This is consistent with the Rosenthal solution [19], where the melt pool temperature during the sintering process increases with increasing laser power when the laser scan speed is kept constant. 


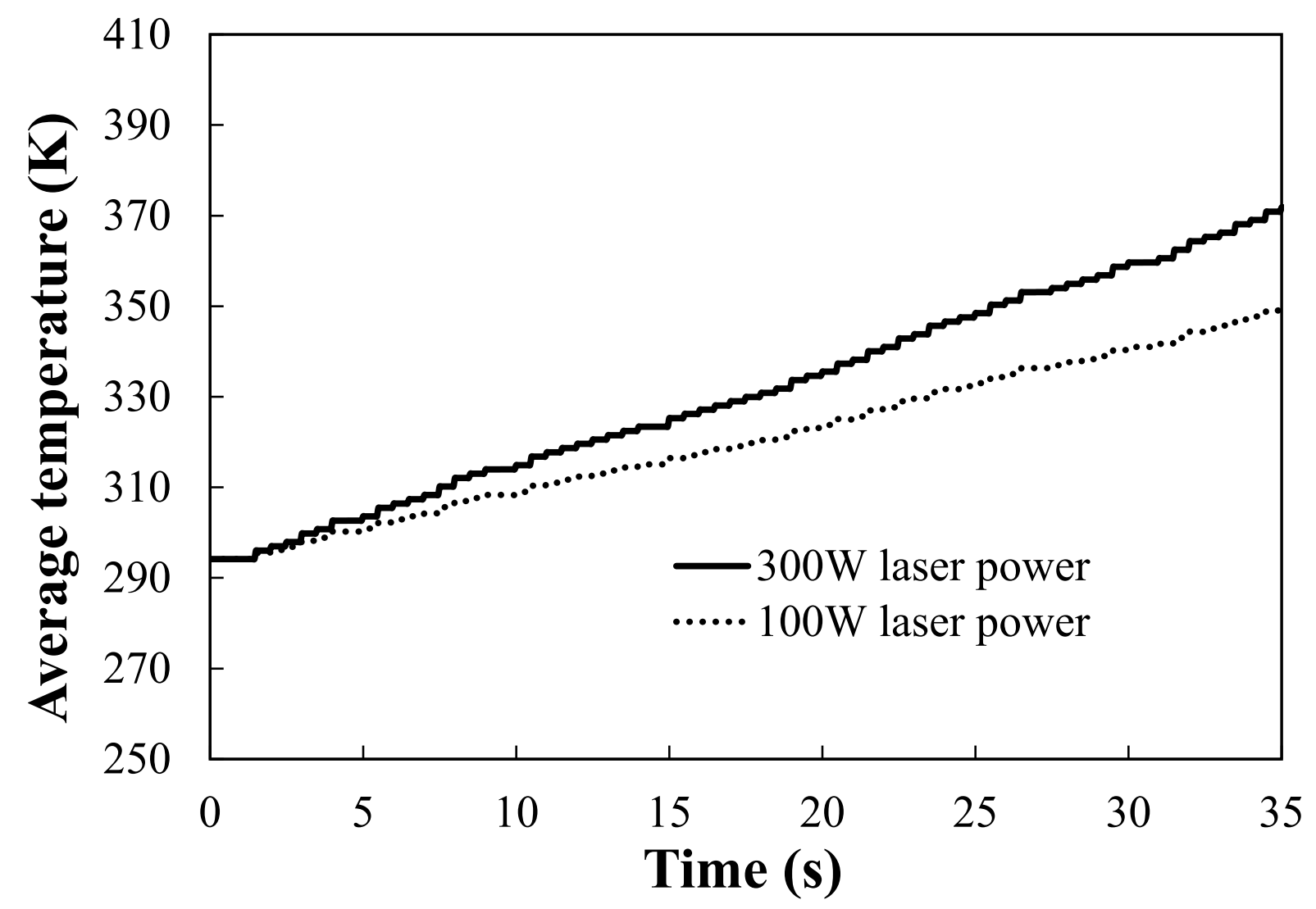

Figure 7: Average temperature of the powder bed versus time for $100 \mathrm{~W}$ and $300 \mathrm{~W}$ laser powers.

3.4 FE modeling of residual stress and distortion of the fabricated component

Using the coupled thermomechanical FE model, the predicted distortion in 3D printed component is shown in Figure 8, compared against experimental observation shown in Figure 8b. The overall shape distortion comparison shows a good agreement. As shown in Figure 8, the distortion is measured at the bottom right of the support material. The predicted distortion from the model is 
$5.59 \mathrm{~mm}$, which is in good agreement with the experimental measurement of $5.22 \mathrm{~mm}$. Additionally, the cracking location is correctly predicted, which is in the middle of support-solid interface.
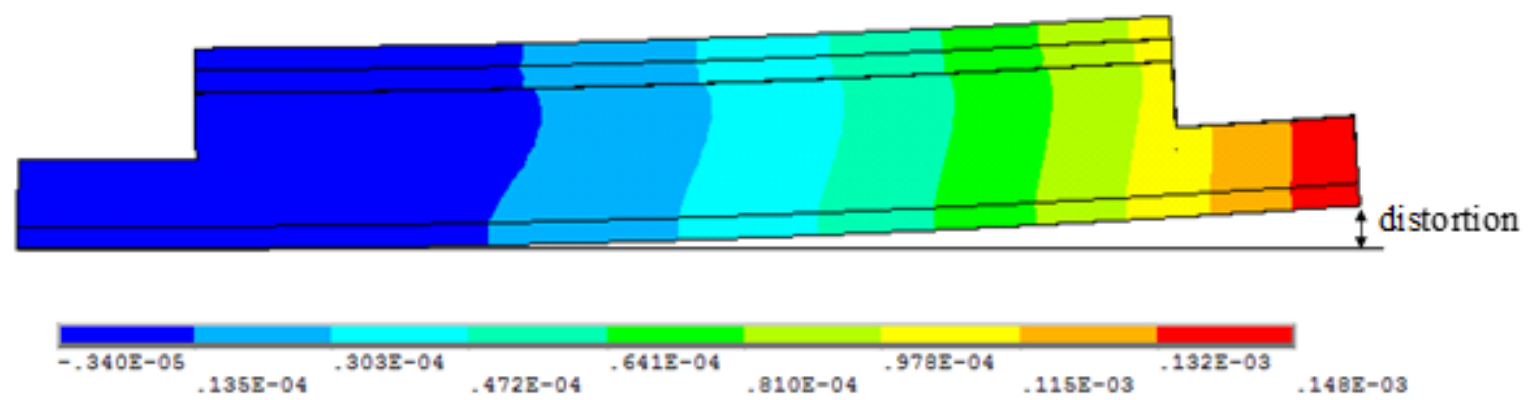

(a)

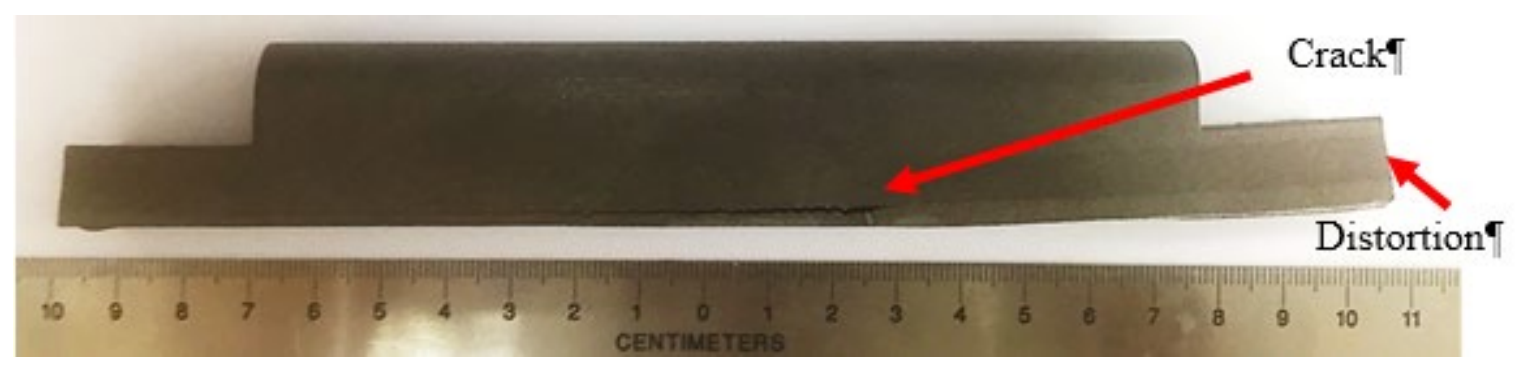

(b)

Figure 82: Comparison of simulation predicted distortion with experimental result. (a) predicted vertical displacement (unit: $\mathrm{m}$ ), (b) experimental observation of distortion of the component. The crack and distortion of the component are also marked.

\section{Concluding remarks}

In this work, a multi-scale multi-physics modeling framework for the L-PBF process is presented. Molecular dynamics model, discrete element, and finite element model were developed to 
understand sintering, powder flow, residual stress and cracking in the components. The coupling of among these models is discussed. The major conclusions are summarized as follows.

1. The diffusion of atoms is higher on particle surface than the particle core. The calculated activation energy of nickel particle diffusion is $6.10 \mathrm{KJ} / \mathrm{mole}$ in the particle core, and 6.24 $\mathrm{KJ} /$ mole on the particle surface, which are reasonably in agreement with experimental data $7.89 \mathrm{KJ} / \mathrm{mole}$.

2. Atomistic tensile test simulations of sintered material show that sintered nickel particles have lower mechanical strengths than the bulk nickel crystal because of their porous structures. Higher heating rate leads to a higher mechanical strength because of accelerated sintering rates.

3. The effect of laser power on the temperature distribution of the powder bed was studied using the DEM. The average temperature in the powder bed increases with higher laser power.

4. The predicted distortion of the L-PBF printed component is in good agreement with experiment.

\section{Acknowledgement}

We acknowledge the financial support provided by the Walmart Foundation (project title: Optimal Plastic Injection Molding Tooling Design and Production through Advanced Additive Manufacturing). Y.G. Jung acknowledges the additional financial supports provided by the Korea Institute of Energy Technology Evaluation and Planning (KETEP) and the Ministry of Trade, Industry \& Energy (MOTIE) of the Republic of Korea (No. 20172020108530), and by "Human Resources Program in Energy Technology" of the KETEP and MOTIE of the Republic of Korea (No. 20174030201460). 


\section{References}

[1] G. Bonny, R. C. Pasianot, and L. Malerba, "Fe-Ni many-body potential for metallurgical applications," Modelling and Simulation in Materials Science and Engineering, vol. 17, Mar 2009.

[2] M. S. Daw and M. I. Baskes, "Embedded-atom method: Derivation and application to impurities, surfaces, and other defects in metals," Physical Review B, vol. 29, pp. 64436453, 1984.

[3] S. Plimpton, "Fast Parallel Algorithms for Short-Range Molecular Dynamics," Journal of Computational Physics, vol. 117, pp. 1-19, 3/1/ 1995.

[4] Y. Zhang, L. Wu, H. El-Mounayri, K. Brand, and J. Zhang, "Molecular Dynamics Study of the Strength of Laser Sintered Iron Nanoparticles," Procedia Manufacturing, vol. 1, pp. 296-307, // 2015.

[5] Y. Zhang and J. Zhang, "Sintering phenomena and mechanical strength of nickel based materials in direct metal laser sintering process - a molecular dynamics study," Journal of Materials Research, vol. 31, pp. 2233-2243, 2016.

[6] A. Manne and N. Satyam, "A Review on the Discrete Element Modeling of Dynamic Laboratory Tests for Liquefaction Assessment," EJGE, vol. 20, 2015.

[7] C. Kloss, C. Goniva, A. Hager, S. Amberger, and S. Pirker, "Models, algorithms and validation for opensource DEM and CFD-DEM," Progress in Computational Fluid Dynamics, an International Journal, vol. 12, pp. 140-152, 2012.

[8] B. Harthong, J. F. Jérier, P. Dorémus, D. Imbault, and F. V. Donzé, "Modeling of highdensity compaction of granular materials by the Discrete Element Method," International Journal of Solids and Structures, vol. 46, pp. 3357-3364, 9// 2009.

[9] E. J. R. Parteli and T. Pöschel, "Particle-based simulation of powder application in additive manufacturing," Powder Technology, vol. 288, pp. 96-102, 1// 2016.

[10] V. Vedachalam, "Discrete Element Modelling Of Granular Snow Particles Using LIGGGHTS," M. Sc. thesis, University of Edinburgh, 2011.

[11] N. V. Brilliantov, F. Spahn, J.-M. Hertzsch, and T. Pöschel, "Model for collisions in granular gases," Physical review E, vol. 53, p. 5382, 1996.

[12] L. E. Silbert, D. Ertaş, G. S. Grest, T. C. Halsey, D. Levine, and S. J. Plimpton, "Granular flow down an inclined plane: Bagnold scaling and rheology," Physical Review E, vol. 64, p. 051302, 2001.

[13] H. Zhang and H. Makse, "Jamming transition in emulsions and granular materials," Physical Review E, vol. 72, p. 011301, 2005.

[14] Y. Zhang and J. Zhang, "Finite element simulation and experimental validation of distortion and cracking failure phenomena in direct metal laser sintering fabricated component," Additive Manufacturing, vol. 16, pp. 49-57, 2017/08/01/ 2017.

[15] S. Sagar, Y. Zhang, L. Wu, H.-Y. Park, J.-H. Lee, and Y.-G. Jung, "Room-Temperature Charpy Impact Property of 3D Printed 15-5 Stainless Steel," Journal of Materials Engineering and Performance, (DOI: 10.1007/s11665-017-3085-9), 2018.

[16] A. G. Elliot and Z. A. Munir, "The sintering of nickel/aluminium spheres to nickel plates," Journal of Materials Science, vol. 3, pp. 150-157, 1968/03/01 1968.

[17] S. Alexander and A. Karsten, "Extracting dislocations and non-dislocation crystal defects from atomistic simulation data," Modelling and Simulation in Materials Science and Engineering, vol. 18, p. 085001, 2010. 
[18] Wikipedia. Nickel. Available: http://en.wikipedia.org/wiki/Nickel

[19] I. Gibson, D. W. Rosen, and B. Stucker, Additive Manufacturing Technologies: 3D Printing, Rapid Prototyping, and Direct Digital Manufacturing: Springer, 2015. 